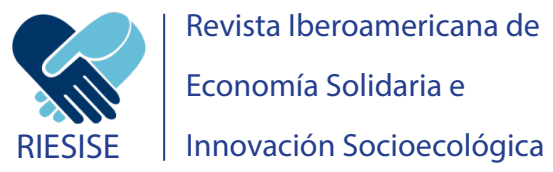

e-ISSN: 2659-3351

Vol. 1 (2018), pp. 11-22

\title{
REFLEXIONES TEÓRICAS SOBRE LA RELACION ENTRE DESARROLLO ENDOGENO Y ECONOMIA SOCIAL
}

\author{
THEORETICAL REFLECTIONS ON THE RELATIONSHIP \\ BETWEEN ENDOGENOUS DEVELOPMENT AND THE SOCIAL \\ ECONOMY \\ Antonio Vázquez Barquero* \\ Universidad Autónoma de Madrid. \\ vazquez-barquero@uam.es
}

\begin{abstract}
Resumen
El desarrollo endógeno y la economía social analizan una realidad compleja, como es el desarrollo de las localidades y territorios. El objeto de este trabajo es mostrar cómo la economía social considera que la solidaridad y la participación ciudadana son clave en los procesos de desarrollo, y cuáles son sus diferencias con el desarrollo local. Pero, dado que la economía social se adapta a la realidad y cambia de unos territorios a otros, contribuye de hecho al desarrollo solidario de los territorios.
\end{abstract}

Palabras Clave

Economía social; desarrollo territorial; cooperativas; iniciativas locales.

\section{Códigos JEL: O10, O15, O18.}

Fecha de recepción: 18/10/2018

Fecha de aceptación: 28/10/2018

Fecha de publicación: 10/12/2018

\section{ABSTRACT}

The endogenous development and the social economy analyze a complex reality, such as the development of localities and territories. The purpose of this paper is to show how the social economy considers that solidarity and citizen participation are key in development processes, and what their differences are with local development. But, given that the social economy adapts to reality and changes from one territory to another, it in fact contributes to the solidarity development of the territories.

\section{KEYWORDS}

Social economy; endogenous development; local initiatives; cooperativism.

\footnotetext{
* El autor agradece a la profesora Maicu Lacalle los comentarios al artículo.
} 


\section{INTRODUCCIÓN}

Desde principios de los años ochenta ha tenido una gran difusión la interpretación del desarrollo endógeno y se ha prestado una creciente atención a la de economía social. Quizás, el mayor atractivo sea su utilidad para comprender los procesos de desarrollo de los territorios y las iniciativas locales en tiempos en los que se producen importantes transformaciones en la economía y la sociedad como consecuencia de la globalización.

Sin duda, se trata de visiones que se utilizan en las ciencias sociales y en las administraciones públicas. Sus aportaciones se apoyan en las críticas a la interpretación que desde la segunda guerra mundial y durante más de cuarenta años, dominó la conceptualización del desarrollo y de las politicas públicas. Dado que las iniciativas locales se basan en las nuevas interpretaciones del desarrollo y de la economía social conviene precisar el alcance y las implicaciones de ambos enfoques.

Este trabajo muestra cómo el desarrollo local y la economía social analizan el desarrollo de los territorios con interpretaciones propias, y responde a preguntas como las siguientes: ¿El desarrollo endógeno es una interpretación territorial? ¿La economía social introduce la solidaridad en la visión del desarrollo? ¿Son importantes sus diferencias con el desarrollo local? ¿Los emprendimientos de las empresas sociales estimulan el desarrollo de las localidades y territorios?

La presentación de este trabajo se hará de la forma siguiente. Se inicia planteando que el concepto de desarrollo evoluciona a medida que lo hace la sociedad. A continuación, se analiza cómo los mecanismos de la economía social activan el bienestar social, y se señala la diversidad de las empresas sociales y las diferencias de los emprendimientos. Después, se muestra en qué factores del desarrollo endógeno la economía social es relevante. El trabajo finaliza señalando las diferencias entre la economía social y el desarrollo local y las aportaciones de la economía social al desarrollo endógeno.

\section{DESARROLLO ENDÓGENO Y PARTICIPACIÓN TERRITORIAL}

A principios de los años ochenta surge la teoría del desarrollo endógeno (Vázquez, 2007). En ella confluyen dos líneas de pensamiento: una, fruto de la búsqueda de una visión que permita actuar para lograr el desarrollo tardío de los territorios (Stöhr, 1981; Friedmann y Douglas, 1978); y la otra, 
que es el resultado del análisis de los procesos de desarrollo industrial en localidades y regiones del sur de Europa (Becattini, 1979; Fua, 1983; Vázquez, 1983). El desarrollo endógeno es una interpretación que considera que las redes de empresas son estratégicas en los sistemas productivos (Johannisson, 1995; Hakansson y Johanson, 1993), que entiende la innovación como un proceso evolutivo (Dosi, 1988; Maillat, 1995), y que reconoce que las instituciones y la cultura condicionan los procesos de desarrollo (North, 1981 y 1986; Ostrom, 1990).

Frecuentemente, se asocia el desarrollo endógeno con la capacidad de las comunidades locales para utilizar el potencial de desarrollo existente en el territorio y responder a los desafíos que se plantean en un momento histórico determinado, como sucede en la actualidad con los efectos de la globalización. Esta visión implica una valoración positiva del potencial de desarrollo, en tanto en cuanto permite a las localidades y territorios dar la respuesta adecuada y satisfacer las necesidades de la población (Vázquez, 1988).

El desarrollo endógeno es una interpretación que analiza cómo las fuerzas del desarrollo generan progreso económico y social (Vázquez, 2002). Sin duda, los factores económicos y productivos condicionan los procesos de acumulación de capital, pero no lo hacen de manera aislada, sino que es la interacción entre ellos lo que impulsa el aumento de la productividad y el crecimiento. Es decir, el desarrollo depende de los efectos que produce la coordinación entre las fuerzas del desarrollo.

De acuerdo con lo anterior, los procesos de crecimiento y cambio estructural de los territorios se dinamizan cuando actúan conjuntamente las fuerzas que activan los procesos de desarrollo, creando sinergias entre ellas y reforzando su efecto sobre los rendimientos del capital y del trabajo. Se puede decir, entonces, que los factores de desarrollo endógeno actúan en red, lo que permite aumentar el efecto de cada uno de ellos en los procesos de desarrollo.

Existen diversos enfoques del desarrollo endógeno. Así, en un momento concreto, una comunidad territorial, por iniciativa propia, puede encontrar nuevas ideas y proyectos que le permitan utilizar sus recursos y dar una respuesta a sus necesidades y desafíos. Así, los actores locales, a través de las iniciativas y decisiones de inversión y de su participación en la formulación y gestión de las políticas, contribuyen al desarrollo productivo de los territorios (Friedmann y Weaber, 1979). Las estrategias de "desarrollo desde abajo", que permiten movilizar y canalizar los recursos y las capacidades existentes en el territorio, impulsan el progreso económico y social cuando las empresas y los actores locales se organizan y realizan sus iniciativas de forma coordinada (Stöhr, 1981).

En este sentido, Arocena y Marsiglia (2017) indican que el desarrollo endógeno es un proceso con dimensiones económicas y sociales. La uti- 
lización de las capacidades, de la tecnología disponible y de las reglas de los territorios impulsan el desarrollo economico local. Las visiones del desarrollo, como el desarrollo autocentrado o el desarrollo participativo, surgen como reacción a la insatisfacción que generó el agotamiento del modelo de desarrollo desde fuera, que fué la interpretación dominante en los años sesenta y setenta. Por ello, la promoción de emprendimientos mediante iniciativas que utilizan los recursos existentes en el territorio, ha adquirido mayor difusión.

Esta interpretación considera que el desarrollo económico y social no es importado, sino que se produce gracias al ahorro y la inversión de las comunidades locales. Para crear empleo y erradicar la pobreza, la estrategia más eficaz sería que el sistema productivo movilizara el potencial de desarrollo existente en el territorio y que la población local participara en el diseño y control de las políticas de desarrollo (Gore, 1984; Kitching, 1982).

\section{ECONOMÍA SOCIAL Y DESARROLLO LOCAL}

Desde principios de los años ochenta, la economía social ha recibido una atención creciente, ya que en las comunidades locales surgen iniciativas basadas en la solidaridad, que utilizan los recursos y potencialidades de que disponen los territorios. La economía social permite superar las diferencias entre capital y trabajo e introducir la solidaridad en el proceso productivo, y propone una visión del desarrollo que incluye a las administraciones públicas, a las empresas privadas y a las organizaciones sociales.

Esta interpretación del desarrollo argumenta que la economía social es la respuesta de la sociedad a las carencias (en el empleo, en la vivienda, en la calidad de vida) que el mercado y el estado no son capaces de superar (Toscano, 2000). Se trata de emprendimientos orientados al bienestar social, que realizan las cooperativas, las micro y pequeñas empresas, las cajas de ahorro, y las organizaciones sin ánimo de lucro, en las que prima el trabajo realizado por quienes participan en la gestión, y en donde las decisiones se toman de forma democrática. Además, la economía social permite integrar a los grupos de población con riesgos de exclusión y convertirlos en actores del desarrollo de los territorios.

El concepto de economía social es complejo, sobre todo porque adquiere formas diferentes según los territorios (Chaves et al. 2013). En el informe para el Comité Europeo, Chaves y Monzón definen la economía social, diferenciando entre las empresas privadas, creadas para satisfacer las necesidades de sus socios a través del mercado y cuyos beneficios se distribuyen entre ellos, de un lado; y las entidades privadas que producen bienes y servicios para los socios y sus familias, y que no se venden en los mercados, de otro. Las cooperativas, las mutuas y las mutualidades de previsión social, y los emprendedores formarían el primer grupo, mientras 
que las asociaciones y fundaciones producirían los bienes y servicios que se distribuyen entre los socios.

El trabajo social se realiza en el entorno territorial formado por las asociaciones y obedece a una cultura específica. Entre sus objetivos se pueden destacar los siguientes: disminuir la desigualdad, fortalecer las capacidades de las personas, mostrar las oportunidades de los grupos sociales para superar los retos a los que se enfrentan, y apoyar a los asociados de manera que consigan mejorar su bienestar.

No existe una única interpretación de la economía social (Chaves, 1999), sino que existen visiones diferentes según los países. En el mundo anglosajón está muy difundida la acepción de organizaciones sin fines de lucro desde los años noventa: organizaciones voluntarias de carácter privado, que controlan sus actividades y que no reparten beneficios entre los socios (Salamon y Anheier, 1997). En Europa e Iberoamérica suelen ser las cooperativas y las asociaciones, en las que domina la reciprocidad entre sus miembros, las que forman la economía solidaria que se unen al mercado y al estado (Laville, 1994). Además, a principios de los años noventa han tomado una fuerza significativa las empresas sociales, que forman las cooperativas, las asociaciones, las mutualidades y las fundaciones (Defourny y Nyssens, 2012).

La globalización está condicionando el papel que juegan las empresas sociales y las organizaciones sin fines de lucro, ya que funcionan como un actor más, juntamente con las empresas privadas y las administraciones públicas. Las organizaciones y las empresas sociales venden sus productos en los mercados y participan en los procesos de redistribución, conectándose con los actores públicos y privados para lo que utilizan los recursos existentes en el territorio (Evers, 1995). Ello puede generar tensiones, ya que las empresas sociales incluyen mecanismos participativos cuando cooperan con las organizaciones públicas y las empresas privadas en las iniciativas de desarrollo.

La diversidad de visiones del pensamiento social, a su vez, genera complejidad al plantear la naturaleza de las actividades productivas. Algunas interpretaciones se centran en la misión social cuando argumentan que la mano de obra de baja cualificación es la que produce los bienes y servicios que se venden en los mercados. Pero, se considera también que las empresas sociales pueden utilizar estrategias innovadoras que permitan satisfacer las necesidades de la población. En estos procesos, el sistema territorial de innovación juega un papel clave, ya que facilita la interacción de los actores locales con las redes de empresas privadas, las administraciones públicas y los actores sociales.

La actividad de las empresas sociales y su dinámica en las localidades y territorios, se estimula cuando el contexto económico, político y cultural facilita la capacidad emprendedora, y, por lo tanto, los emprendimientos sociales. 
Ello explica la complejidad conceptual, la especificidad de las iniciativas locales, y la diversidad de las estrategias y de las politicas de desarrollo.

En resumen, la visión social del desarrollo endógeno sostiene que, en la actualidad, lo importante del desarrollo es su carácter autónomo, basado en la utilización de los recursos propios y que, por lo tanto, se puede producir en localidades y territorios que disponen de potencial de desarrollo. La cuestión estaría en utilizar los recursos locales en emprendimientos y actividades en cuyo diseño y control participasen los ciudadanos y las organizaciones locales, lo que les permitiría controlar el proceso a través de las iniciativas locales.

\section{LA RELEVANCIA DE LA ECONOMÍA SOCIAL EN EL DESARROLLO EN- DÓGENO}

La conceptualización del desarrollo endógeno y de la economía social tal como se acaba de plantear, muestra que ambas visiones tienen un carácter territorial y entienden que las respuestas a los retos son más eficaces cuando se realizan "de abajo hacia arriba". Además, a pesar de las diferencias asociadas con la interpretación del desarrollo, comparten mecanismos importantes en los procesos de desarrollo (Janssen et al., 2018).

Gráfico N 1. Desarrollo endógeno: factores explicativos. Fuente: Vázquez Barquero (2002).

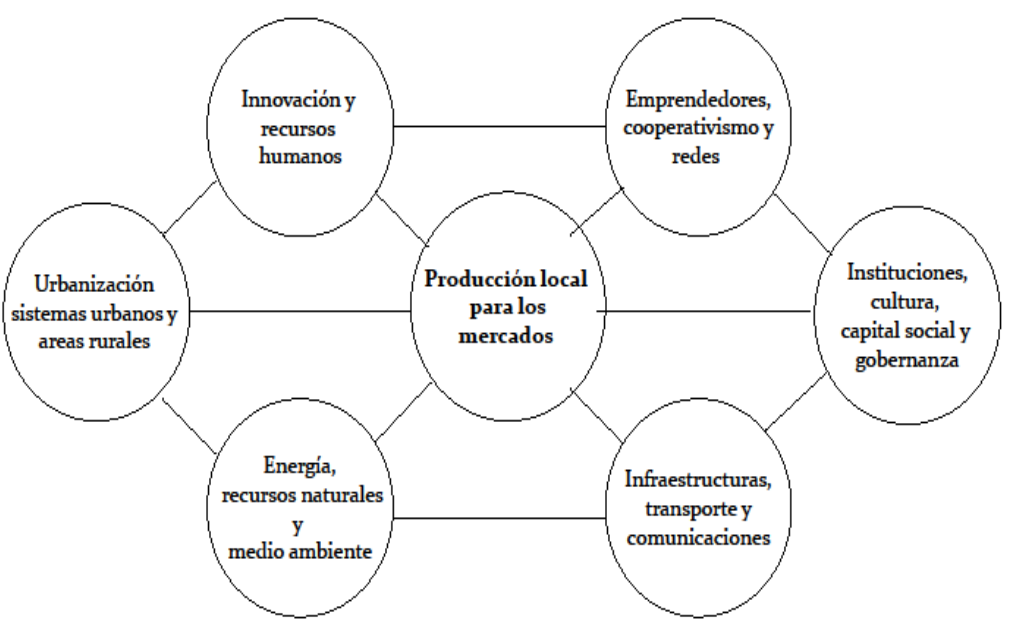

Una de las fuerzas centrales del desarrollo endógeno hace referencia a la capacidad emprendedora y al funcionamiento de los sistemas productivos locales (Vázquez, 2002). Tanto en la economía social como en el desarrollo local se acepta la importancia de las redes y de los sistemas flexibles de 
empresas, así como la interacción de los actores locales, lo que permite generar economías en los sistemas productivos locales. En la economía social, las redes de empresas se apoyan en los sistemas de relaciones prexistentes entre los actores locales. Además, el cooperativismo se ha difundido durante los últimos años en los sistemas productivos locales (Lacalle, 2002). Pero, hasta ahora en la economía social no se consideran las redes nacionales de empresas ni las cadenas globales de valor.

La disponibilidad de recursos humanos es clave en los procesos de desarrollo, y en ambas interpretaciones se les da importancia, sin duda en lo que atañe a la actividad productiva; si bien la formación no se considera estratégica en la economía social. Quizás un elemento diferencial de ambas interpretaciones sea la importancia que se le concede a las empresas innovadoras, a la maquinaria, y al desarrollo de la rebotica en el desarrollo local. En todo caso, también la economía social reconoce que la innovación es importante y que los sistemas territoriales de innovación son clave en la creación y difusión del conocimiento, por lo que cuando los actores interactúan, las empresas sociales contribuyen a la evolución de las innovaciones (Nicholls, 2009).

La visión del desarrollo local señala que la geografía cuenta ya que, durante las últimas décadas, tanto en los sistemas urbanos como en las áreas rurales se ha producido una importante diferenciación productiva (Porter et al. 2004; Sassen, 2007). Algunas ciudades se han convertido en espacios preferentes de las nuevas actividades industriales y de servicios, mientras que las localidades y territorios rurales se desarrollan diferenciando sus producciones lo que les permite ampliar las ventas de sus productos en los mercados nacionales e internacionales. Han surgido, así, las ciudades creativas, las ciudades lentas, las regiones agroindustriales y los territorios reserva de los recursos naturales, que son importantes en ambas visiones.

Los procesos de desarrollo tienen profundas raíces institucionales y culturales, aunque no siempre se valoran en la economía social. Los sistemas productivos se apoyan en las reglas de juego formales e informales, que condicionan su funcionamiento. Dorado y Ventresca (2013) señalan que el entorno institucional facilita que las empresas sociales aprovechen las oportunidades que se les presentan. Pero, el entorno institucional puede ser favorable o perjudicial, sobre todo cuando las empresas sociales realizan actividades interregionales. Las reglas y la cultura de los territorios condicionan el funcionamiento de los emprendimientos y la confianza de los actores económicos, de manera que el sistema institucional puede alterar la lógica en la toma de decisiones e influir en las iniciativas de las empresas sociales.

Con la globalización el sistema de transporte y comunicaciones se está transformando (Shabani y Safaie, 2018). Por ello, dado que los intercambios 
de bienes y servicios están condicionados por las infraestructuras terrestres, marítimas y aéreas, su funcionamiento afecta al desarrollo economico y social, sobre todo en los territorios de desarrollo tardío. Es más, en la actualidad las comunicaciones se han ampliado en todo tipo de territorios gracias a la difusión de internet y a la disponibilidad de teléfonos móviles, lo que le ha dado un nuevo impulso a la economía social y al desarrollo local.

La energía juega, también, un papel relevante en la actividad económica de los territorios y sus resultados dependen del sistema productivo y de las políticas públicas (Dubach, 2002; Giampietro et al., 2012). Las actividades de la industria eléctrica (generación, transmisión, distribución y comercialización de la electricidad) pueden ser de carácter público como los modelos energéticos integrados verticalmente, o pueden adoptar un modelo privado. Además de que el marco regulador es importante, también lo es el papel que desempeñan los organismos reguladores, cuando es preciso controlar los efectos de la privatización de algunas de las fases del proceso de producción y de la comercialización de la energía eléctrica. Por último, el estado juega un papel clave en el impulso de las energías renovables en el momento actual.

\section{COMENTARIOS FINALES}

La discusión anterior plantea que el desarrollo local y la economía social son interpretaciones que facilitan el análisis del desarrollo endógeno de los territorios. Adoptan una visión de abajo hacia arriba para impulsar los procesos de desarrollo y otorgan un papel estratégico a los actores locales en la realización de los emprendimientos y las iniciativas locales. Sin embargo, entre ellas existen diferencias.

La economía social es una interpretación de los procesos de desarrollo, que considera que las necesidades de la población estarían mejor cubiertas y el éxito de las iniciativas locales garantizado cuando la población define y controla los proyectos, aunque sean limitados los recursos de que se dispone y las inversiones que se realizan. Por lo tanto, la economía social sostiene que el desarrollo se apoya en la acción ciudadana, por lo que las políticas públicas serían más eficientes cuando se diseñan y se gestionan de abajo hacia arriba y cuando las decisiones las toman los ciudadanos en función de sus necesidades.

La economía social tiene también diferencias con el desarrollo local. Ante todo, no considera que sea preciso relacionar el crecimiento y el cambio estructural con la acumulación de capital; ni reconoce siempre la importancia del ahorro y la inversión en los procesos de desarrollo de los territorios; ni recomienda actuar combinando los mecanismos que facilitan la sostenibilidad económica, social y cultural. Además, no sostiene que las instituciones son clave en los procesos de desarrollo, a pesar de que afec- 
tan a las actividades de las empresas sociales y al funcionamiento de los mercados. Por último, la economía social no considera la interacción en las redes de empresas y su integración en los sistemas productivos nacionales e internacionales.

Pero, la economía social, en la práctica, contribuye, de forma importante, al desarrollo endógeno cuando se activan las iniciativas locales. Entre sus aportaciones se pueden destacar las siguientes: la disponibilidad de recursos humanos solidarios, la capacidad innovadora en algunos espacios rurales y urbanos, los emprendimientos sociales, incluyendo los que forman parte de la economía informal, la diferenciación de la producción local que facilite la ampliación de los mercados. Por lo tanto, las empresas sociales contribuyen al desarrollo solidario, cuando crean puestos de trabajo y luchan contra la exclusión social.

\section{BIBLIOGRAFÍA}

Arocena, J. y J. Marsiglia (2017) : La escena territorial del desarrollo. Actores, relatos y politicas. Montevideo, Taurus.

Becattini, G. (1979) :"Dal settore industriale al distretto industriale: alcune considerazione sull'unita di indagine dell'economia industriale". Rivista di Economia e Politica Industriale 1: 7-21.

Chaves, R. (1999): La economia social como enfoque metodológico, como objeto de estudio y como disciplina científica. Ciriec-España, Revista de Economía Pública, Social y Cooperativa 33: 115-140.

Chaves, R. Monzon, J. L. y G. Zaragoza (2013): La economia social: concepto, macromagnitudes y yacimientos de empleo para el Trabajo Social. Cuadernos de Trabajo Social 26 (1): 19-29.

Defourny, J. y M. Nyssens (2012): "El enfoque EMES de empresa social desde una perspectiva comparada". Ciriec-España, Revista de Economía Pública, Social y Cooperativa 75: 6-34.

Dorado, S. y Ventresca, M.J. (2013): "Crescive Entrepreneurship in Complex Social Problems: Institutional Conditions for Entrepreneurial Engagement". Journal of Business Venturing 28 (1): 69-82.

Dosi, G. (1988): "Sources, procedures and microeconomic effects of innovation". Journal of Economic Literature 36: 1126-1171.

Dubach, K. N. (ed.) (2002): Equity and Environment in Electricity Reform. Washington D. C., World Resources Institute.

Evers, A. (1995): "Part of the Welfare Mix: The Third sector as an intermediate area". Voluntas 6 (2): 119-139.

Friedmann, J. y Douglas, M.J. (1978): "Agropolitan development: toward a new strategy for regional planning in Asia", en F. Lo y K. Salih (eds.) Growth Pole Strategy and Regional Planning Development Policy. Oxford, Pergamon. 
Friedmann, J. y Weaver (1979): Territory and Function. Londres, Edward Arnold. Fuá, G. (1983): "L'industrializzazione nel nord est e nel centro", en G. Fuá y C. Zachia (eds.) Industrializzazione senza fratture. Bologna, II Mulino.

Giampietro, M., Mayumi, K. y Sorman, A.H. (2012): The Metabolic Pattern of Societies. Londres, Routledge.

Gore, Ch. (1984): Regions in Question. Space, Development Theory and Regional Policy. Londres, Methuen.

Hakansson, H. y J. Johanson (1993): The network as a governance structure. Interfirm cooperation beyond markets and hierarchies, en G. Grabher (ed.) The Embedde Firm On the Socioeconomics of Industrial Networks. Londres, Routledge.

Janssen, F., Fayolle, A. y Wuilaume, A. (2018): "Researching Bricolage in Social Entrepreneurship". Entrepreneurship and Regional Development 30 (3-4): 450-470.

Johannisson, B. (1995): "Paradigms and entrepreneurial networks - some methodological challenges". Entrepreneurship and Regional Development 7: 215-231.

Kitchin, G. N. (1982): Development and Underdevelopment in Historical Perspective: Populism, Nationalism and Industrialization. Londres, Methuen.

Lacalle, M.C. (2002): Microcréditos. De pobres a microempresarios. Barcelona, Ariel

Laville, J.L. (1994) L"Economie Solidaire. Paris, Desclee de Brouwer.

Maillat, D. (1995): "Territorial dynamic, innovative milieus and regional policy". Entrepreneurship \& Regional Development 7: 157-165.

Nicholls, A. (2009): We do Good Things, don't We?: Blended Value Accounting in Social Entrepreuneurship. Accounting, Organizations and Society 34 (6): 550-568.

North, D.C. (1981): Structure and Change in Economic History. Nueva York, W. W. Norton.

North, D.C. (1986): "The new institutional economics". Journal of Institutional and Theoretical Economics 142 (2): 230-237.

Ostrom, E. (1990): Governing the Commons: The Evolution of Institutions for Collective Actions. New York, Cambridge University Press.

Porter, E. M., Ketels, C. H. M., Miller, K. and R. T. Bryden (2004): Competitiveness in Rural U.S. Regions: Learning and Research Agenda. Report. Boston, Institute for Strategy and Competitiveness, Harvard Business School.

Shabani, Z.D. y Safaie, S. (2018): "Do transport infrastructure spillovers matter for economic growth? Evidence on road and railway transport in Iranian Provinces". Regional Science, Police and Practice 5 (2): 201-218.

Salamon, L. M. y Anheier, H.K. (1997): Defining the non-profit sector: a cross-national analysis. Baltimore, Institute for Policy Studies, The Hopkins University. 
Sassen, S. (2007): "El reposicionamiento de las ciudades y regiones urbanas en una economía global". Revista Eure 100: 9-34.

Stöhr, W. B. (1981): "Development from below: the bottom-up and periphery inward development paradigm", en W. B. Stöhr and D. R. Taylor (eds.) Development from Above or Below? Chichester, J. Wiley and Sons.

Toscano Sánchez, F. (2000): "Desarrollo Local y Economía Social", en Pérez Ramírez, B. y Carrillo Benito, E. (eds.) Desarrollo Local: Manual de Uso. Madrid, ESIC Editorial.

Vázquez Barquero, A. (1983): Industrialization in Rural Areas. The Spanish Case, Report, OECD meeting, Senigallia, July 7-10, CT/RUR/113/06, OECD.

Vázquez Barquero, A. (1988): Desarrollo local, una estrategia de creación de empleo. Madrid, Pirámide.

Vázquez Barquero, A. (2002): Endogenous Development: Networking, innovation, institutions and cities. Londres, Routledge.

Vázquez Barquero, A. (2007): Desarrollo endógeno. Teorías y políticas de desarrollo territorial. Investigaciones Regionales, 11: 183-210. 